\title{
CERTAIN RESULTS ON SUBORDINATIONS ASSOCIATED WITH A DIFFERINTEGRAL OPERATOR
}

\author{
JACEK DZIOK, RAVINDER KRISHNA RAINA, JANUSZ SOKÓŁ
}

\begin{abstract}
We investigate some interesting properties on subordinations for classes of multivalent analytic functions associated with a linear differintegral operator of J. Dziok [3]. Some applications and important consequences of the main results are also discussed.
\end{abstract}

\section{Introduction and preliminaries}

Let $\mathscr{A}(p)(p \in \mathrm{N})$ denote the class of functions of the form

$$
f(z)=z^{p}+\sum_{n=1}^{\infty} a_{n+p} z^{n+p}=\sum_{n=p}^{\infty} a_{n} z^{n} \quad\left(a_{p}=1\right),
$$

which are analytic and $p$-valent in the open unit disc $\mathscr{U}=\{z:|z|<1\}$ on the complex plane C. For two analytic functions $f, g \in \mathscr{A}(p)$, we say that $f$ is subordinate to $g$, written as $f \prec g$, if and only if there exists a Schwarz function $\omega$ (with $\omega(0)=0$ and $|\omega(z)|<1$ ) analytic in $\mathcal{U}$ such that $f(z)=g(\omega(z)$ ). In particular, if $g$ is univalent in $\mathscr{U}$, then we have the following equivalence:

$$
f(z) \prec g(z) \Longleftrightarrow f(0)=g(0) \text { and } f(|z|<1) \subset g(|z|<1) .
$$

The concept of subordination was mainly used in defining various classes of functions and studying their important properties in the geometric function theory. We recall here the following two classes defined by

$$
\mathscr{S}_{p}^{*}[\varphi]:=\left\{f \in \mathscr{A}(p): \frac{z f^{\prime}(z)}{f(z)} \prec \varphi(z), z \in \mathscr{U}\right\}
$$

and

$$
\mathscr{K}_{p}[\varphi]:=\left\{f \in \mathscr{A}(p):\left[1+\frac{z f^{\prime \prime}(z)}{f^{\prime}(z)}\right] \prec \varphi(z), z \in \mathcal{U}\right\},
$$

Received 15 June 2010. 
where $\varphi$ is analytic in $\mathscr{U}$ with $\varphi(0)=p$. For $p=1$ and $\varphi(z)=\frac{1+z}{1-z}$, we obtain the well known classes $\mathscr{S}^{*}$ and $\mathscr{K}$ of starlike and convex functions, respectively. Also, by choosing the function $\varphi$ suitably in (3) and (4), we obtain several other function classes which have been investigated in recent past ([14], [15]). If we restrict our attention to the functions $\varphi$ which map $\mathscr{U}$ onto a disc or a half-plane, then we obtain the classes

$$
\mathscr{S}_{p}^{*}\left[p \frac{1+A z}{1+B z}\right] \quad(-1 \leq B<A \leq 1)
$$

and

$$
\mathscr{K}_{p}\left[p \frac{1+A z}{1+B z}\right] \quad(-1 \leq B<A \leq 1),
$$

which were introduced and investigated (for $p=1$ ) by Janowski [5]. Moreover, for $p=1, B=-1$ and $A=1-2 \alpha$, the above classes (5) and (6) become the classes $\mathscr{S}_{1}^{*}\left[\frac{1+(1-2 \alpha) z}{1-z}\right]=\mathscr{S}^{*}(\alpha)$ and $\mathscr{K}_{1}\left[\frac{1+(1-2 \alpha) z}{1-z}\right]=\mathscr{K}(\alpha)$ of $\alpha$-starlike and $\alpha$-convex functions, respectively, which were introduced by Robertson [10].

It is interesting to consider the conditions joining $z f^{\prime}(z) / f(z)$ with $z f^{\prime \prime}(z) / f^{\prime}(z)$, and indeed, Mocanu introduced a new class $\mathcal{M}(\alpha)(\alpha \in \mathrm{R})$ in [6] defined by

$$
\begin{aligned}
& \mathscr{M}(\alpha):=\left\{f \in \mathscr{A}:\left[\alpha\left(1+\frac{z f^{\prime \prime}(z)}{f^{\prime}(z)}\right)\right.\right. \\
& \left.\left.+(1-\alpha) \frac{z f^{\prime}(z)}{f(z)}\right] \prec \frac{1+z}{1-z} \text { for } z \in \mathcal{U}\right\},
\end{aligned}
$$

which provides a continuous passage from the convex to starlike functions.

In this paper we prove certain results giving the subordination properties for classes of functions which are multivalently analytic in the open unit disk, and associated with a differintegral operator (defined below). Some interesting consequences and applications are also considered.

Let us consider the Dziok differintegral operator $\Omega_{\beta}^{\alpha}$ (introduced in [3]) which for the purpose of this paper is defined here for $f \in \mathscr{A}(p)$ as follows:

$$
\Omega_{\beta}^{\alpha} f(z)=\frac{z^{1-\alpha-\beta}}{\Gamma(\alpha)} \int_{0}^{z}(z-\xi)^{\alpha-1} \xi^{\beta-1} f(\xi) \mathrm{d} \xi
$$

$(\Re \alpha>0, \Re \beta>-p)$,

$$
\Omega_{\beta}^{\alpha} f(z)=\frac{z^{1-\alpha-\beta}}{\Gamma(1+\alpha)} \frac{\mathrm{d}}{\mathrm{d} z} \int_{0}^{z}(z-\xi)^{\alpha} \xi^{\beta-1} f(\xi) \mathrm{d} \xi
$$


$(-1<\Re \alpha \leq 0, \Re \beta>-p)$,

$$
\Omega_{\beta}^{\alpha} f(z)=z^{1-\alpha-\beta} \frac{\mathrm{d}^{m}}{\mathrm{~d} z^{m}} \frac{\Omega_{\beta}^{\gamma} f(z)}{z^{1-\alpha-\beta-m}}
$$

$(\Re \alpha \leq-1, \alpha=\gamma-m,-1<\Re \gamma \leq 0, \Re \beta>-p, m \in \mathrm{N})$.

The multiplicities of $(z-\xi)^{\alpha-1}$ and $(z-\xi)^{\gamma}$ are removed by requiring that $\log (z-\xi) \in \mathrm{R}$ for $z-\xi>0$. If $f(z) \in \mathscr{A}_{p}$ is given by (1), then we can write (in terms of gamma function)

$$
\begin{aligned}
\Omega_{\beta}^{\alpha} f(z) & =\sum_{n=p}^{\infty} \frac{\Gamma(n+\beta) a_{n}}{\Gamma(n+\beta+\alpha)} z^{n} \\
& = \begin{cases}\frac{\Gamma(\beta)}{\Gamma(\beta+\alpha)} \sum_{n=p}^{\infty} \frac{(\beta)_{n} a_{n}}{(\beta+\alpha)_{n}} z^{n} & \text { for } \alpha+\beta \neq 0 \\
\Gamma(\beta) \sum_{n=p}^{\infty} \frac{(\beta)_{n} a_{n}}{(n-1) !} z^{n} & \text { for } \alpha+\beta=0\end{cases} \\
& = \begin{cases}\frac{\Gamma(\beta)}{\Gamma(\beta+\alpha)}\left[\sum_{n=p}^{\infty} \frac{(\beta)_{n}}{(\beta+\alpha)_{n}} z^{n} * \sum_{n=p}^{\infty} a_{n} z^{n}\right] & \text { for } \alpha+\beta \neq 0 \\
\Gamma(\beta)\left[\sum_{n=p}^{\infty} \frac{(\beta)_{n}}{(n-1) !} z^{n} * \sum_{n=p}^{\infty} a_{n} z^{n}\right] & \text { for } \alpha+\beta=0\end{cases}
\end{aligned}
$$

where $(\beta)_{n}$ is the familiar Pochhammer symbol defined by

$$
(\beta)_{n}=\frac{\Gamma(\beta+n)}{\Gamma(\beta)}= \begin{cases}1 & \text { for } n=0, \beta \neq 0 \\ \beta(\beta+1) \ldots(\beta+n-1) & \text { for } k \in \mathrm{N}=\{1,2,3, \ldots\},\end{cases}
$$

and $*$ denotes the Hadamard product given by

$$
\left[\sum_{n=p}^{\infty} a_{n} z^{n}\right] *\left[\sum_{n=p}^{\infty} b_{n} z^{n}\right]=\left[\sum_{n=p}^{\infty} a_{n} b_{n} z^{n}\right] .
$$

Ruscheweyh [11] introduced an operator $\mathscr{D}^{\lambda}: \mathscr{A}(1) \rightarrow \mathscr{A}(1)(\lambda \geq-1)$ such that

$$
\mathscr{D}^{\lambda} f(z)=\frac{z}{(1-z)^{\lambda+1}} * f(z)=z+\sum_{n=1}^{\infty} \frac{(\lambda+1)_{n}}{n !} a_{n+1} z^{n+1},
$$

which implies that for $\lambda=n \in \mathrm{N} \cup\{0\}$ :

$$
\mathscr{D}^{n} f(z)=\frac{z\left(z^{n-1} f(z)\right)^{(n)}}{n !},
$$

and $\mathscr{D}^{n} f(z)$ is then called the Ruscheweyh differential operator. It may be observed from (8) and (9) that for $f \in \mathscr{A}(p)$, we have the relationship:

$$
z^{p-1} \mathscr{D}^{\lambda}\left[z^{1-p} f(z)\right]=\frac{1}{\Gamma(\lambda+1)} \Omega_{1+\lambda}^{-\lambda} f(z) .
$$


Next, we recall the generalized Bernardi integral operator $\mathscr{J}_{\beta, v}: \mathscr{A}(1) \rightarrow$ $\mathscr{A}(1)$, which is defined by (cf. [1])

$$
\mathscr{J}_{\beta, \nu}[f(z)]=\frac{v+\beta}{z^{\nu}} \int_{0}^{z} t^{\nu-1} f^{\beta}(t) \mathrm{d} t \quad(\beta, v \in \mathbf{C}),
$$

and for $\beta=1$, the operator (10) becomes the Bernardi operator which on operating to the function $f(z)=z+a_{2} z^{2}+a_{3} z^{3}+\cdots$, gives

$$
\mathscr{J}_{1, v}[f(z)]=\sum_{n=1}^{\infty} \frac{v+1}{v+n} a_{n} z^{n} .
$$

The Bernardi operator becomes the Livingston operator [9] (or the Libera operator [8]) for special choices of $\beta$ and $v$. We also note that for $f \in \mathscr{A}(p)$, the operators (8) and (10) are connected to each other by the following relationship:

$$
z^{p-1} \mathscr{J}_{1, v}\left[z^{1-p} f(z)\right]=\frac{1+v}{v}(v)_{n} \Omega_{v}^{1} f(z) .
$$

Using the definitions of fractional calculus, Srivastava and Owa [15] (see also [2], [13], [14]) have defined the linear operator $\widehat{\Omega}^{\lambda}: \mathscr{A}(1) \rightarrow \mathscr{A}(1)$ by

$$
\widehat{\Omega}^{\lambda} f(z)=\Gamma(2-\lambda) z^{\lambda} D_{z}^{\lambda} f(z) \quad(\lambda \neq 2,3,4, \ldots),
$$

where

$$
D_{z}^{\lambda} f(z)= \begin{cases}\frac{1}{\Gamma(1-\lambda)} \frac{\mathrm{d}}{\mathrm{d} z} \int_{0}^{z} \frac{f(\zeta)}{(z-\zeta)^{\lambda}} \mathrm{d} \zeta & (0 \leq \lambda<1), \\ \frac{\mathrm{d}^{\mathrm{n}}}{\mathrm{d} \mathrm{z}^{\mathrm{n}}} D_{z}^{\lambda-n} f(z) & (n \leq \lambda<n+1 ; n=0,1,2, \ldots),\end{cases}
$$

and the multiplicity of $(z-\zeta)^{\lambda}$ is removed by requiring $\log (z-\zeta)$ to be real when $z-\zeta>0$. It is easy to observe for $f \in \mathscr{A}(p)$ that

$$
z^{p-1} \widehat{\Omega}^{\lambda}\left[z^{1-p} f(z)\right]=(1-\lambda) \Gamma(1-\lambda) \Omega_{1-\lambda}^{1} f(z) .
$$

Following the idea of a Mocanu class $\mathscr{M}(\alpha)$, let us consider for $\sigma \in[0,1]$ and $h(z)=1+a_{1} z+a_{2} z^{2}+\cdots$, a function class $\mathscr{J}\left(\sigma, \Omega_{\beta}^{\alpha} ; h\right)$ defined by

$$
\begin{aligned}
\mathscr{J}\left(\sigma, \Omega_{\beta}^{\alpha} ; h\right):=\{f \in \mathscr{A}(p) & : \sigma\left[1+\frac{z\left(\Omega_{\beta}^{\alpha} f(z)\right)^{\prime \prime}}{\left(\Omega_{\beta}^{\alpha} f(z)\right)^{\prime}}\right] \\
& \left.+(1-\sigma) \frac{z\left(\Omega_{\beta}^{\alpha} f(z)\right)^{\prime}}{\Omega_{\beta}^{\alpha} f(z)} \prec p h(z), z \in \mathcal{U}\right\},
\end{aligned}
$$


and in this paper we shall mainly focus our investigations on the particular class

$$
\mathscr{J}^{*}\left(\sigma, \Omega_{\beta}^{\alpha} ; A, B\right):=\mathscr{J}\left(\sigma, \Omega_{\beta}^{\alpha} ; \frac{1+A z}{1+B z}\right),
$$

where $A, B \in \mathrm{C},|B| \leq 1$. The classes $\mathscr{J}^{*}\left(\sigma, \Omega_{\beta}^{\alpha} ; A, B\right), 0 \leq \sigma \leq 1$ give a continuous passage from $\mathscr{J}^{*}\left(0, \Omega_{\beta}^{\alpha} ; A, B\right)$ to $\mathscr{J}^{*}\left(1, \Omega_{\beta}^{\alpha} ; A, B\right)$. Notice that for $A=-B=1$, the class $\mathscr{J}^{*}\left(0, \Omega_{\beta}^{\alpha} ; 1,-1\right)$ becomes the class $G_{p}(\alpha, \beta)$ which was earlier considered in [3], and $\mathscr{J}\left(\sigma, \Omega_{\beta}^{0} ; 1,-1\right)=\mathscr{M}(\sigma)$, where $\mathscr{M}(\sigma)$ is the Mocanu class (7).

After some calculations, we obtain from (8):

$$
\frac{z\left(\Omega_{\beta}^{\alpha} f(z)\right)^{\prime}}{\Omega_{\beta}^{\alpha} f(z)}=\frac{\Omega_{\beta}^{\alpha-1} f(z)}{\Omega_{\beta}^{\alpha} f(z)}+1-(\alpha+\beta)
$$

and

$$
1+\frac{z\left(\Omega_{\beta}^{\alpha} f(z)\right)^{\prime \prime}}{\left(\Omega_{\beta}^{\alpha} f(z)\right)^{\prime}}=\frac{\left(\Omega_{\beta}^{\alpha-1} f(z)\right)^{\prime}}{\left(\Omega_{\beta}^{\alpha} f(z)\right)^{\prime}}+1-(\alpha+\beta),
$$

and therefore, the class (11) becomes

$$
\begin{aligned}
\mathscr{J}\left(\sigma, \Omega_{\beta}^{\alpha} ; h\right)=\{f \in & \mathscr{A}(p): \sigma\left[\frac{\left(\Omega_{\beta}^{\alpha-1} f(z)\right)^{\prime}}{\left(\Omega_{\beta}^{\alpha} f(z)\right)^{\prime}}\right] \\
& \left.+(1-\sigma) \frac{\Omega_{\beta}^{\alpha-1} f(z)}{\Omega_{\beta}^{\alpha} f(z)}+1-(\alpha+\beta) \prec p h(z)\right\}
\end{aligned}
$$

\section{Main results}

It follows easily that the functions $h(z)=1+a_{1} z+a_{2} z^{2}+\cdots$ and $F(z)=$ $c_{p} z^{p}+c_{p+1} z^{p+1}+\cdots$ (which are analytic in $\mathscr{U}$ ) satisfying the subordination relation

$$
\sigma\left[1+\frac{z F^{\prime \prime}(z)}{F^{\prime}(z)}\right]+(1-\sigma) \frac{z F^{\prime}(z)}{F(z)} \prec p h(z) \quad(z \in \mathscr{U})
$$

can be rewritten as the Briot-Bouquet type differential subordination in the form

$$
\phi(z)+\frac{\sigma z \phi^{\prime}(z)}{p \phi(z)} \prec h(z) \quad(z \in \mathcal{U})
$$


where

$$
\phi(z)=\frac{z F^{\prime}(z)}{p F(z)}=1+b_{1} z+\cdots \quad(z \in \mathscr{U}) .
$$

Evidently, the subordination in (11) is of the Briot-Bouquet type differential subordination, and we can write

$$
\mathscr{J}\left(\sigma, \Omega_{\beta}^{\alpha} ; h\right)=\left\{f \in \mathscr{A}(p): s(z)+\frac{\sigma z s^{\prime}(z)}{p s(z)} \prec h(z), z \in \mathscr{U}\right\},
$$

where

$$
s(z)=\frac{z\left[\Omega_{\beta}^{\alpha} f(z)\right]^{\prime}}{p \Omega_{\beta}^{\alpha} f(z)}=1+d_{1} z+\cdots \quad(z \in \mathcal{U}) .
$$

One of the basic results in the theory of Briot-Bouquet differential subordinations is a theorem given in [4], which states (as its particular case) that if $h$ is convex univalent with positive real part in $\mathcal{U}$ and the functions $\phi, h$ satisfy (16) with real $\sigma$ and $p$, then $\phi(z) \prec h(z)$. Hence, on using (18), we directly get the following result.

THEOREM 1. Let $h$ be convex univalent with positive real part in $\mathscr{U}$ and $f \in \mathscr{J}\left(\sigma, \Omega_{\beta}^{\alpha} ; h\right)$, then

$$
\frac{z\left[\Omega_{\beta}^{\alpha} f(z)\right]^{\prime}}{p \Omega_{\beta}^{\alpha} f(z)} \prec h(z) \quad(z \in \mathscr{U}) .
$$

COROLlary 1. Under the assumptions of Theorem $1, \mathscr{J}\left(\sigma, \Omega_{\beta}^{\alpha} ; h\right) \subset$ $\mathscr{J}\left(0, \Omega_{\beta}^{\alpha} ; h\right)$ for all $\sigma \in(0,1]$.

Let $f$ be given by (1). If we denote

$$
\begin{aligned}
\theta(z) & =\frac{p z^{1-p} \Omega_{\beta}^{\alpha} f(z)}{\left[\Omega_{\beta}^{\alpha} f\right]^{\prime}(0)} \\
& =\frac{\Gamma(\beta+\alpha+p)}{\Gamma(\beta+p)} \sum_{k=1}^{\infty} \frac{\Gamma(k+\beta+p-1)}{\Gamma(k+\beta+\alpha+p-1)} a_{k+p-1} z^{k} \\
& =\frac{\beta+\alpha+p-1}{\beta+p-1} \sum_{k=1}^{\infty} \frac{(\beta+p-1)_{k}}{(\beta+\alpha+p-1)_{k}} a_{k+p-1} z^{k} \quad(z \in \mathcal{U}),
\end{aligned}
$$

then we can express (20) in the form

$$
\frac{z \theta^{\prime}(z)+(p-1) \theta(z)}{p \theta(z)} \prec h(z) \quad(z \in \mathscr{U}) .
$$


Thus for $h(z)=\frac{1+A z}{1+B z},|B| \leq 1$, (22) gives

$$
\frac{z \theta^{\prime}(z)}{\theta(z)} \prec p \frac{1+A z}{1+B z}+1-p=\frac{1+z(p(A-B)+B)}{1+B z} \quad(z \in \mathscr{U})
$$

and therefore, we have proved the following corollary.

Corollary 2. Suppose that $|B| \leq 1$. Let a function $f$ of the form (1) belong to the class $\mathscr{J}^{*}\left(\sigma, \Omega_{\beta}^{\alpha} ; A, B\right)$, then the function $\theta(z)$ defined by (21) satisfies the subordination (23). This means that $\theta$ belongs to the class

$$
\mathscr{S}_{1}^{*}\left[\frac{1+z(p(A-B)+B)}{1+B z}\right],
$$

where $S_{1}^{*}$ is defined by (5).

We observe here that the above Theorem 1 can effectively be made applicable leading to some interesting consequences, whenever, we know more about the function $h$. If $h$ is a special bilinear transformation a result more appealing than (20) can be derived by employing the results from [7]. After some adaptations, Theorem 3.2j [7, p. 97] becomes the following lemma.

Lemma 1 ([7]). Suppose that $\beta>0$ and $n$ is a positive integer. Let us denote

$$
\mathscr{R}_{\beta, n}(z)=\beta \frac{1+z}{1-z}+\frac{2 n z}{1-z^{2}} \quad(z \in \mathscr{U}),
$$

and let $h$ be a convex univalent function in $\mathscr{U}$, with $h(0)=1$ such that

$$
\beta h(z) \prec \mathscr{R}_{\beta, n}(z) \quad(z \in \mathscr{U}),
$$

then the Briot-Bouquet differential equation

$$
q(z)+\frac{n z q^{\prime}(z)}{\beta q(z)}=h(z) \quad(z \in \mathscr{U})
$$

has a univalent solution $q_{n}$ analytic in $\mathcal{U}$. Furthermore, if the functions $h$ and $\tau(z)=1+a_{n} z^{n}+a_{n+1} z^{n+1}+\cdots$ satisfy the Briot-Bouquet differential subordination

$$
\tau(z)+\frac{z \tau^{\prime}(z)}{\beta \tau(z)} \prec h(z) \quad(z \in \mathscr{U}),
$$

then

$$
\tau(z) \prec q_{n}(z) \prec h(z) \quad(z \in \mathscr{U}),
$$


and the function $q_{n}$ is the best dominant of the subordination (25) in the sense that if there exists a function $p$ such that $\tau(z) \prec p(z) \prec h(z)$, then $q_{n}(z) \prec$ $p(z)$.

Notice that the function $\mathscr{R}_{\beta, n}$ is called the open door function and it is univalent in $\mathscr{U}, \mathscr{R}_{\beta, n}(0)=\beta$. In order to verify (24), it is sufficient (in view of the definition of subordination and (2)) to show that $h(0)=1$ and $\beta h(\mathscr{U}) \subset$ $\mathscr{R}_{\beta, n}(\mathscr{U})$. The set $\mathscr{R}_{\beta, n}(\mathscr{U})$ is the complex plane with slits along the half-lines $\Re w=0$ and $|\Im w| \geq \gamma=n \sqrt{1+2 \beta / n}$, see Fig. 1 below.

Theorem 2. Suppose that $A, B \in \mathrm{C},|B| \leq 1, \sigma \in(0,1], p \in \mathrm{N}$, and let one of the following conditions be satisfied:

(i) $B=e^{i \varphi}, A=r e^{i \varphi}$ for some $r \in(-1, \infty) \backslash\{1\}, \varphi \in \mathrm{R}$,

(ii) $|B|<1$ and $|\Im[A \bar{B}]| \geq\left(1-|B|^{2}\right) \sqrt{1+2 p / \sigma}$ and $\Re[1-A \bar{B}] \geq$ $|A-B|$,

(iii) $|B|<1$ and $|\Im[A \bar{B}]|<\left(1-|B|^{2}\right) \sqrt{1+2 p / \sigma}$ and $(\Re[1-A \bar{B}])^{2}+$ $\left(|\Im[A \bar{B}]|-\frac{\sigma}{p}\left(1-|B|^{2}\right) \sqrt{1+2 p / \sigma}\right)^{2} \geq|A-B|^{2}$.

If $f(z)=a_{p} z^{p}+a_{p+1} z^{p+1}+\cdots$ belongs to the class $\mathscr{J}^{*}\left(\sigma, \Omega_{\beta}^{\alpha} ; A, B\right)$, then the function (19) satisfies

$$
s(z)=\frac{z\left[\Omega_{\beta}^{\alpha} f(z)\right]^{\prime}}{p \Omega_{\beta}^{\alpha} f(z)} \prec \frac{\sigma}{p g_{p}(z)} \prec \frac{1+A z}{1+B z} \quad(z \in \mathscr{U}),
$$

where

$$
g_{p}(z)= \begin{cases}\int_{0}^{1}\left[\frac{1+B t z}{1+B z}\right]^{\frac{p}{\sigma}\left(\frac{A}{B}-1\right)} t^{\frac{p}{\sigma}-1} \mathrm{~d} t, & \text { if } B \neq 0 \\ \int_{0}^{1} e^{\frac{p A(t-1) z}{\sigma}} t^{\frac{p}{\sigma}-1} \mathrm{~d} t, & \text { if } B=0,\end{cases}
$$

and the function $\frac{\sigma}{p g_{p}(z)}$ is the best dominant of the subordination (26).

Proof. To prove Theorem 2, we first apply Lemma 1 (when $\beta=p / \sigma$, $n=1$ and $h(z)=(1+A z) /(1+B z),|B| \leq 1)$, then (24) becomes

$$
\frac{p}{\sigma}\left(\frac{1+A z}{1+B z}\right) \prec \mathscr{R}_{p / \sigma, 1}(z)=\frac{p}{\sigma}\left(\frac{1+z}{1-z}\right)+\frac{2 z}{1-z^{2}} \quad(z \in \mathscr{U}) .
$$

We will try to find $A$ and $B$ such that (28) is satisfied for given values of $p$ and $\sigma$. The right-hand side of (28) is the open door function which is univalent in $\mathcal{U}$ and to verify (28), it is sufficient to show that

$$
\frac{p}{\sigma} h(\mathscr{U}) \subset \mathscr{R}_{p / \sigma, 1}(\mathscr{U}) .
$$




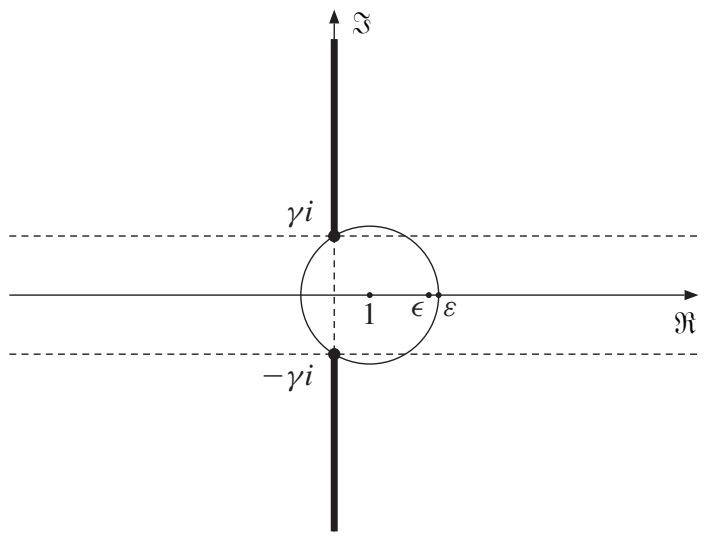

$$
\begin{aligned}
& n=1, \quad p=2, \quad \sigma=\frac{1}{2}, \quad \gamma=3, \quad \frac{p}{\sigma}=4, \quad A=\frac{-13}{4 \sqrt{10}}, \quad \frac{p}{\sigma} C=1, \\
& \epsilon=\frac{p}{\sigma}=4, \quad B=\frac{-3}{4 \sqrt{10}}, \quad \frac{p}{\sigma} R=\sqrt{10}, \quad \varepsilon=1+\sqrt{10}
\end{aligned}
$$

FIGURE 1. $\mathscr{R}_{\beta, n}(\mathscr{U})$

The set $\mathscr{R}_{p / \sigma, 1}(\mathscr{U})$ is the complex plane with two slits, see Fig. 1. For $|B|=1$, $A \neq B$, the set $h(\mathcal{U})$ is the half-plane bounded by

$$
\partial h(\mathscr{U})=\left\{w \in \mathrm{C}: w=\frac{1+A / B}{2}+\frac{1-A / B}{2} i t, t \in \mathrm{R}\right\},
$$

and hence in this case (29) is satisfied, whenever $\partial h(\mathscr{U})$ is parallel to the imaginary axis and on the right side of it. This implies that

$$
|B|=1, \quad A \neq B, \quad \Im\left[\frac{1-A / B}{2}\right]=0 \quad \text { and } \quad \Re\left[\frac{1+A / B}{2}\right] \geq 0,
$$

and elementary calculations in (31) give the condition (i) stated above.

The problem of finding $A$ and $B$ such that (28) is satisfied is rather more complicated when $|B|<1$. We note that in this case the function $h(z)=$ $(1+A z) /(1+B z)$ maps the open unit disc $\mathscr{U}$ onto the disc

$$
D(C, R)=\{w \in C:|w-C|<R\},
$$

where

$$
C=\frac{1-A \bar{B}}{1-|B|^{2}}, \quad R=\frac{|A-B|}{1-|B|^{2}}
$$


To verify (29), we must find $C$ and $R$ such that the disc $\frac{p}{\sigma} D(C, R)$ contains $p / \sigma$ and lies on the right half-plane, or that the $\operatorname{disc} \frac{p}{\sigma} D(C, R)$ contains $p / \sigma$ and also contains a portion of imaginary axis outside the slits $\Re w=0$ and $|\Im w| \geq \gamma=\sqrt{1+2 p / \sigma}$, which means that $\frac{p}{\sigma} D(C, R)$ lies partially on the open door, see Fig. 1 .

It all depends on the position of the center $C$ and on the length of the radius $R$ given in (32). If

$$
\left|\Im\left[\frac{p}{\sigma} C\right]\right| \geq \gamma=\sqrt{1+2 p / \sigma},
$$

then the center $p C / \sigma$ lies outside of the horizontal strip between $\pm \gamma i$ and (29) is satisfied, whenever,

$$
\Re\left[\frac{p}{\sigma} C\right] \geq \frac{p}{\sigma} R .
$$

The requirements $|B|<1$, (33) and (34) yield the desired condition (ii). If

$$
\left|\Im\left[\frac{p}{\sigma} C\right]\right|<\gamma=\sqrt{1+2 p / \sigma},
$$

then the center $p C / \sigma$ lies within the horizontal strip between $\pm \gamma i$ and (29) is satisfied under slightly weaker conditions than mentioned in (ii). In this case, the disc $\frac{p}{\sigma} D(C, R)$ can lie a little on the line segment from $-\gamma i$ to $\gamma i$. Hence, the requirements

$$
\left|\frac{p}{\sigma} C-\gamma i\right| \geq \frac{p}{\sigma} R \quad \text { and } \quad\left|\frac{p}{\sigma} C+\gamma i\right| \geq \frac{p}{\sigma} R
$$

are sufficient for (29) in this case, and these constraints (36) in conjunction with the conditions $|B|<1$ and (35) give the conditions (iii).

If we consider $\gamma \in[0, p)(p \in \mathrm{N})$ and $f \in \mathscr{J}^{*}\left(\sigma, \Omega_{\beta}^{\alpha} ; A, B\right)$ with $A=$ $1-\frac{2 \gamma}{p}, B=-1$, then the condition (i) is satisfied, and Theorem 2 gives

$$
\frac{z\left[\Omega_{\beta}^{\alpha} f(z)\right]^{\prime}}{p \Omega_{\beta}^{\alpha} f(z)} \prec \frac{\sigma}{p g_{p}(z)} \prec \frac{1+\left(1-\frac{2 \gamma}{p}\right) z}{1-z} \quad(z \in \mathscr{U}),
$$

where

$$
g_{p}(z)=\int_{0}^{1}\left[\frac{1-t z}{1-z}\right]^{\frac{2(\gamma-1)}{\sigma}} t^{\frac{p}{\sigma}-1} \mathrm{~d} t
$$


From the subordination (37), we find that

$$
\Re\left[\frac{z\left[\Omega_{\beta}^{\alpha} f(z)\right]^{\prime}}{\Omega_{\beta}^{\alpha} f(z)}\right]>\gamma \quad(0 \leq \gamma<p, z \in \mathscr{U}),
$$

and we obtain the following result.

Corollary 3. Let $0 \leq \gamma<p$ and $f \in \mathscr{J}^{*}\left(\sigma, \Omega_{\beta}^{\alpha} ; A, B\right)$ (with $A=1-\frac{2 \gamma}{p}$, $B=-1)$, then the function

$$
h(z)=\frac{p \Omega_{\beta}^{\alpha} f(z)}{\left[\Omega_{\beta}^{\alpha} f\right]^{\prime}(0)} \quad(z \in \mathscr{U})
$$

is a p-valently starlike function of order $\gamma$.

Using the following integral representation and the identity for the Gaussian hypergeometric function (see, for example [7, p. 7]):

$$
\int_{0}^{1} t^{b-1}(1-t)^{c-b-1}(1-z t)^{-a} \mathrm{~d} t=\frac{\Gamma(b) \Gamma(c-b)}{\Gamma(c)}{ }_{2} F_{1}(a, b, c ; z)
$$

$(\mathfrak{R} c>\mathfrak{R} b>0)$ and

$$
{ }_{2} F_{1}(a, b ; c ; z)=(1-z)^{-a}{ }_{2} F_{1}\left(a, c-b ; c ; \frac{z}{z-1}\right),
$$

we can express the best dominant (38) of the subordination (37) in terms of the hypergeometric function given by

$$
g_{p}(z)=\int_{0}^{1}\left[\frac{1-t z}{1-z}\right]^{\frac{2(\gamma-1)}{\sigma}} t^{\frac{p}{\sigma}-1} \mathrm{~d} t=\frac{\sigma}{p}{ }_{2} F_{1}\left(\frac{2(1-\gamma)}{\sigma}, 1,1+\frac{p}{\sigma} ; \frac{z}{z-1}\right) .
$$

It can be verified that the sharp order of $p$-valently starlikeness is $\min \left\{\Re\left[g_{p}(z)\right]:|z|<1\right\}$, and for the details that it is $g_{p}(-1)$, one may refer to [7, pp. 112-114].

THEOREM 3. Let $h$ be a convex function and $0 \leq \sigma_{1} \leq \sigma_{2} \leq 1$, then

$$
\mathscr{J}\left(\sigma_{2}, \Omega_{\beta}^{\alpha} ; h\right) \subset \mathscr{J}\left(\sigma_{1}, \Omega_{\beta}^{\alpha} ; h\right)
$$

Proof. If $\sigma_{1}=0$, then Corollary 1 readily follows (39). Suppose now that $\sigma_{1}>0, f \in \mathscr{J}\left(\sigma_{2}, \Omega_{\beta}^{\alpha} ; h\right)$ and $s$ is given by (19). Then $\frac{\sigma_{1}}{\sigma_{2}} \in(0,1]$, and

$$
s(z)+\frac{\sigma_{1} z s^{\prime}(z)}{p s(z)}=\frac{\sigma_{1}}{\sigma_{2}}\left(s(z)+\frac{\sigma_{2} z s^{\prime}(z)}{p s(z)}\right)+\left(1-\frac{\sigma_{1}}{\sigma_{2}}\right) s(z) \prec h(z),
$$


because in view of (18), (20) and (40), there exists a convex combination of functions subordinated to the convex function $h$, and so this combination is subordinated to $h$ too. Therefore $f \in \mathscr{J}\left(\sigma_{1}, \Omega_{\beta}^{\alpha} ; h\right)$.

Lemma 2. Suppose that $f \in \mathscr{J}^{*}\left(\sigma, \Omega_{\beta}^{\alpha} ; A, B\right)$, and let one of the following conditions be satisfied

(j) $B=e^{i \varphi}, A=r e^{i \varphi}$ for some $r \in\left(1-\frac{2}{p}, \infty\right) \backslash\{1\}, \varphi \in \mathrm{R}$,

(jj) $|B|<1$ and $\Re[1-[p(A-B)+B] \bar{B}] \geq p|A-B|$.

Then the function $\theta(z)=p z^{1-p} \Omega_{\beta}^{\alpha} f(z) /\left[\Omega_{\beta}^{\alpha} f\right]^{\prime}(0)$ given in (21) belongs to the class $\mathscr{S}^{*}$ of starlike functions.

Proof. In view of Corollary 2, the function $\theta$ given by (21) satisfies (23), and hence $\theta$ is a starlike function, whenever, the function

$$
g^{*}(z)=\frac{1+z(p(A-B)+B)}{1+B z} \quad(z \in \mathscr{U})
$$

satisfies $\Re\left[g^{*}(z)\right]>0(z \in \mathscr{U})$. If $|B|=1$, then the set $g^{*}(\mathscr{U})$ is a halfplane. Repeating similar consideration as given in the first part of the proof of Theorem 2, we infer that the conditions $|B|=1, A \neq B$,

$$
\Im\left[\frac{1-[p(A-B)+B] / B}{2}\right]=0
$$

and

$$
\mathfrak{R}\left[\frac{1+[p(A-B)+B] / B}{2}\right] \geq 0
$$

are equivalent to $\Re\left[g^{*}(z)\right]>0$. After some calculations, these requirements become the conditions (j). Next for $|B|<1$, the set $g^{*}(\mathscr{U})$ is a disc, and in this case $\Re\left[g^{*}(z)\right]>0$ if and only if the real part of its center is greater than or equal to the length of its radius. Using (32) suitably, we get

$$
\Re\left[\frac{1-[p(A-B)+B] \bar{B}}{1-|B|^{2}}\right] \geq\left|\frac{p(A-B)}{1-|B|^{2}}\right|,
$$

which yields the conditions (jj).

Theorem 4. Let $p \in \mathrm{N}$. Suppose that A and B satisfy (j) or (jj) given in Lemma 2. If $\alpha$ and $\beta$ are such that the function

(41) $\tilde{H}(z)$

$$
=\frac{(p+\beta+\alpha)(1+p+\beta+\alpha)}{p} \sum_{k=1}^{\infty} \frac{(k+p-1) z^{k}}{(k+p-1+\beta+\alpha)(k+p+\beta+\alpha)}
$$


belongs to the class $\mathscr{K}$ of convex functions, then

$$
\mathscr{J}^{*}\left(\sigma, \Omega_{\beta}^{\alpha} ; A, B\right) \subset \mathscr{J}^{*}\left(\sigma, \Omega_{\beta}^{\alpha+2} ; A, B\right)
$$

Proof. To prove Theorem 4, we take advantage of the several convolution results due mainly to Ruscheweyh [11], [12]. Let $f \in \mathscr{J}^{*}\left(\sigma, \Omega_{\beta}^{\alpha} ; A, B\right)$, then it easily follows that

$$
\Omega_{\beta}^{\alpha+2} f(z)=g(z) * \Omega_{\beta}^{\alpha} f(z)
$$

where

$$
g(z)=\sum_{n=p}^{\infty} \frac{z^{n}}{(n+\beta+\alpha)(n+\beta+\alpha+1)} \quad(z \in \mathscr{U})
$$

From (43) and (44), we obtain

$$
\begin{aligned}
& \sigma\left[1+\frac{z\left(\Omega_{\beta}^{\alpha+2} f(z)\right)^{\prime \prime}}{\left(\Omega_{\beta}^{\alpha+2} f(z)\right)^{\prime}}\right]+(1-\sigma) \frac{z\left(\Omega_{\beta}^{\alpha+2} f(z)\right)^{\prime}}{\Omega_{\beta}^{\alpha+2} f(z)} \\
& =\sigma\left[1+\frac{z\left(\Omega_{\beta}^{\alpha} f * g\right)^{\prime \prime}(z)}{\left(\Omega_{\beta}^{\alpha} f * g\right)^{\prime}(z)}\right]+(1-\sigma) \frac{z\left(\Omega_{\beta}^{\alpha} f * g\right)^{\prime}(z)}{\left(\Omega_{\beta}^{\alpha} f * g\right)(z)} \\
& =\sigma \frac{z g^{\prime}(z) * z\left(\Omega_{\beta}^{\alpha} f\right)^{\prime}(z)}{z g^{\prime}(z) * \Omega_{\beta}^{\alpha} f(z)}+(1-\sigma) \frac{g(z) * z\left(\Omega_{\beta}^{\alpha} f\right)^{\prime}(z)}{g(z) * \Omega_{\beta}^{\alpha} f(z)} \\
& =\sigma\left[\frac{z^{2-p} g^{\prime}(z) * z\left(z^{1-p} \Omega_{\beta}^{\alpha} f\right)^{\prime}(z)}{z^{2-p} g^{\prime}(z) * z^{1-p} \Omega_{\beta}^{\alpha} f(z)}-(1-p)\right] \\
& +(1-\sigma)\left[\frac{z^{1-p} g(z) * z\left(z^{1-p} \Omega_{\beta}^{\alpha} f\right)^{\prime}(z)}{z^{1-p} g(z) * z^{1-p} \Omega_{\beta}^{\alpha} f(z)}-(1-p)\right] \\
& =\sigma \frac{z^{2-p} g^{\prime}(z) * z\left(z^{1-p} \Omega_{\beta}^{\alpha} f\right)^{\prime}(z)}{z^{2-p} g^{\prime}(z) * z^{1-p} \Omega_{\beta}^{\alpha} f(z)} \\
& +(1-\sigma) \frac{z^{1-p} g(z) * z\left(z^{1-p} \Omega_{\beta}^{\alpha} f\right)^{\prime}(z)}{z^{1-p} g(z) * z^{1-p} \Omega_{\beta}^{\alpha} f(z)}-(1-p) \\
& =\sigma \frac{\widetilde{H}(z) * z \theta^{\prime}(z)}{\widetilde{H}(z) * \theta(z)}+(1-\sigma) \frac{\frac{p z^{1-p} g(z)}{g^{\prime}(0)} * z \theta^{\prime}(z)}{\frac{p z^{1-p} g(z)}{g^{\prime}(0)} * \theta(z)}-(1-p),
\end{aligned}
$$


where $\theta$ is given by (21). For $\Re x \geq 0$, or $x=0$, the function

$$
\tilde{h}(x ; z)=\sum_{k=1}^{\infty} \frac{(1+x) z^{k}}{(k+x)}
$$

is convex univalent ([11]), and in the famous paper [12], it was proved as a result of the Pólya-Schoenberg conjecture that the functions which are convex univalent have the same property preserved under a convolution structure also. Accordingly, under our assumptions on $\alpha, \beta$ and $p$, the function

$$
\begin{aligned}
\widetilde{g}(z) & =\frac{p z^{1-p} g(z)}{g^{\prime}(0)}=\sum_{k=1}^{\infty} \frac{(p+\beta+\alpha)(1+p+\beta+\alpha)}{(k+p-1+\beta+\alpha)(k+p+\beta+\alpha)} z^{k} \\
& =\left[\sum_{k=1}^{\infty} \frac{p+\beta+\alpha}{k+p-1+\beta+\alpha} z^{k}\right] *\left[\sum_{k=1}^{\infty} \frac{1+p+\beta+\alpha}{k+p+\beta+\alpha} z^{k}\right] \quad(z \in \mathscr{U})
\end{aligned}
$$

is in $\mathscr{K}$ (since in view of (45), it is expressed as the convolution of two functions belonging to $\mathscr{K}$ ). From (23), we have

$$
z \theta^{\prime}(z)=\theta(z)\left[p \frac{1+A \omega(z)}{1+B \omega(z)}+1-p\right]:=h(z)\left[q_{A, B}(\omega(z))+1-p\right],
$$

where $\omega$ is an analytic function with $\omega(0)=0$ and $|\omega(z)|<1$ for $z \in \mathscr{U}$. Using (47) and (48) in (45), we obtain

$$
\begin{aligned}
\sigma[1+ & \left.\frac{z\left(\Omega_{\beta}^{\alpha+2} f(z)\right)^{\prime \prime}}{\left(\Omega_{\beta}^{\alpha+2} f(z)\right)^{\prime}}\right]+(1-\sigma) \frac{z\left(\Omega_{\beta}^{\alpha+2} f(z)\right)^{\prime}}{\Omega_{\beta}^{\alpha+2} f(z)} \\
& =\sigma \frac{\widetilde{H}(z) * \theta(z) q_{A, B}(\omega(z))}{\widetilde{H}(z) * \theta(z)}+(1-\sigma) \frac{\widetilde{g}(z) * \theta(z) q_{A, B}(\omega(z))}{\widetilde{g}(z) * \theta(z)},
\end{aligned}
$$

In the paper [12], it was also proved that if $q(z)=1+b_{1} z+\cdots, f \in \mathscr{K}$ and $g \in \mathscr{S}^{*}$, then

$$
\frac{f(z) * q(z) g(z)}{f(z) * g(z)} \in \overline{\operatorname{co}} q(\mathscr{U}) \quad(z \in \mathcal{U}),
$$

where $\overline{\mathrm{co}} q(\mathcal{U})$ denotes the closed convex hull of the set $q(\mathscr{U})$. By our assumptions that $\widetilde{H} \in \mathscr{K}, \widetilde{g} \in \mathscr{K}$ (in view of (47)) and $h \in \mathscr{S}^{*}$ (in view of Lemma 2), we therefore infer from (50) (on appealing to (49)) that we have a convex combination of values of $\overline{\mathrm{co}} q_{A, B}(\omega(\mathscr{U}))$. Since $\overline{\mathrm{co}} q_{A, B}(\omega(\mathscr{U})) \subset \overline{\mathrm{co}} q_{A, B}(\mathscr{U})$, 
where $q_{A, B}(z)$ is a convex univalent function, we deduce from (2) that

$$
\left[1+\frac{z\left(\Omega_{\beta}^{\alpha+2} f(z)\right)^{\prime \prime}}{\left(\Omega_{\beta}^{\alpha+2} f(z)\right)^{\prime}}\right]+(1-\sigma) \frac{z\left(\Omega_{\beta}^{\alpha+2} f(z)\right)^{\prime}}{\Omega_{\beta}^{\alpha+2} f(z)} \prec p \frac{1+A z}{1+B z},
$$

which implies that $f \in \mathscr{J}^{*}\left(\sigma, \Omega_{\beta}^{\alpha+2} ; A, B\right)$.

On setting

$$
\alpha+\beta=-1, \quad p \geq 2
$$

in Theorem 4, then the function (41) becomes

$$
\widetilde{H}(z)=\sum_{k=1}^{\infty} \frac{(1+p-2) z^{k}}{k+p-2} \quad(z \in \mathcal{U}),
$$

and in view of (46) it belongs to the class of convex function $\mathscr{K}$. We thus get the following result.

Corollary 4. Let $\beta+\alpha=-1$ and $p \geq 2$, then

$$
\mathscr{J}^{*}\left(\sigma, \Omega_{\beta}^{\alpha} ; A, B\right) \subset \mathscr{J}^{*}\left(\sigma, \Omega_{\beta}^{\alpha+2} ; A, B\right) .
$$

Next, if we set

$$
\alpha+\beta=0, \quad p \geq 1
$$

in Theorem 4, then the function (41) becomes

$$
\widetilde{H}(z)=(1+p) \sum_{k=1}^{\infty} \frac{z^{k}}{k+p} \quad(z \in \mathscr{U}),
$$

and in view of (46) it belongs to the class of convex functions $\mathscr{K}$, and we get the following result.

Corollary 5. Let $\beta+\alpha=0$ and $p \geq 1$, then

$$
\mathscr{J}^{*}\left(\sigma, \Omega_{\beta}^{\alpha} ; A, B\right) \subset \mathscr{J}^{*}\left(\sigma, \Omega_{\beta}^{\alpha+2} ; A, B\right) .
$$

\section{REFERENCES}

1. Bernardi, S. D., Convex and starlike univalent functions, Trans. Amer. Math. Soc. 135 (1969), 429-446.

2. Dziok, J., Classes of analytic functions involving some integral operator, Folia Sci. Univ. Tech. Resoviensis 20 (1995), 21-39.

3. Dziok, J., Applications of the Jack lemma, Acta Math. Hungar. 105 (2004), 93-102. 
4. Eenigenburg, P., Miller, S. S., Mocanu, P. T., and Reade, M. O., On a Briot-Bouquet differential subordination, pp. 1-13 in: Seminar of Geometric Function Theory, Preprint 82-4, Univ. Babeş -Bolyai, Cluj-Napoca 1983.

5. Janowski, W., Extremal problems for a family of functions with positive real part and for some related families, Ann. Polon. Math. 23 (1970), 159-177.

6. Mocanu, P. T., Une propriété de convexité généralisée dans la théorie de la représentation conforme, Mathematica (Cluj) 11(34) (1969), 127-133.

7. Miller, S. S.,and Mocanu, P. T., Differential Subordinations. Theory and Applications, Monographs and Textbooks in Pure and Appl. Math. 225, Dekker, New York 2000.

8. Libera, R. J., Some classes of regular univalent functions, Proc. Amer. Math. Soc. 16 (1965), 755-758.

9. Livingston, A. E., On the radius of univalence of certain analytic functions, Proc. Amer. Math. Soc. 17 (1966), 352-357.

10. Robertson, M. S., Certain classes of starlike functions, Michigan Math. J. 32 (1985), 135-140.

11. Rusheweyh, St., New criteria for univalent functions, Proc. Amer. Math. Soc. 49 (1975), 109-115.

12. Ruscheweyh, St., and Sheil-Small, T., Hadamard product of schlicht functions and the PólyaSchoenberg conjecture, Comment. Math. Helv. 48 (1973), 119-135.

13. Srivastava, H. M., and Aouf, M. K., A certain fractional derivative operator and its applications to a new class of analytic and multivalent functions with negative coefficients I and II, J. Math. Anal. Appl. 171 (1992), 1-13 and 192 (1995), 673-688.

14. Srivastava, H. M., and Owa, S., (eds.), Current Topics in Analytic Function Theory, World Scientific, Singapore 1992.

15. Srivastava, H. M., and Owa, S., (eds.), Univalent Functions, Fractional Calculus, and Their Applications, Proc. Kōriyama 1988, Halsted Press, New York 1989.

INSTITUTE OF MATHEMATICS

UNIVERSITY OF RZESZÓW

UL. REJTANA 16A

35-310 RZESZÓW

POLAND

E-mail: jdziok@univ.rzeszow.pl

M.P. UNIVERSITY OF AGRI. AND TECHNOLOGY

UDAIPUR

RAJASTHAN

INDIA

PRESENT ADDRESS:

10/11 GANPATI VIHAR

OPPOSITE SECTOR 5

UDAIPUR 313002

INDIA

E-mail: rkraina_7@hotmail.com
DEPARTMENT OF MATHEMATICS RZESZÓW UNIVERSITY OF TECHNOLOGY UL. W. POLA 2 35-959 RZESZÓW POLAND

E-mail: jsokol@prz.edu.pl 